\title{
SOFTWARE PLATFORM FOR RAPID PROTOTYPING OF NIRS BRAIN COMPUTER INTERFACING TECHNIQUES
}

\author{
Fiachra Matthews ${ }^{23} \quad$ Christopher Soraghan ${ }^{1} \quad$ Tomas E. Ward ${ }^{4}$ \\ Charles Markham $^{3}$ Barak A. Pearlmutter ${ }^{23}$ \\ ${ }^{1}$ Experimental Physics $\quad{ }^{2}$ Hamilton Institute $\quad{ }^{3}$ Computer Science $\quad{ }^{4}$ Electronic Engineering \\ National University of Ireland Maynooth, Co. Kildare, Ireland
}

\begin{abstract}
This paper describes the control system of a nextgeneration optical brain-computer interface (BCI). Using functional near-infrared spectroscopy (fNIRS) as a BCI modality is a relatively new concept, and research has only begun to explore approaches for its implementation. It is necessary to have a system by which it is possible to investigate the signal processing and classification techniques available in the BCI community. Most importantly, these techniques must be easily testable in real-time applications. The system we describe was built using LABVIEW, a graphical programming language designed for interaction with National Instruments hardware. This platform allows complete configurability from hardware control and regulation, testing and filtering in a graphical interface environment.
\end{abstract}

\section{INTRODUCTION}

This paper outlines a software tool for use in an fNIRS based BCI. This section will describe briefly the background to BCI and principals behind fNIRS. Section II will take a software engineering approach to the necessary requirements of a control structure for such a device. It will outline the hardware involved and how the current software meets the requirements.

\section{I-A. Brain-Computer Interfacing \& fNIRS}

Brain-Computer Interfaces (BCIs) attempt to use physiological correlates of volitional mental activity to provide an alternative communication channel for a user to interact with the outside world. fNIRS, pioneered in [1], uses an optical window of tissue transparency in the near-infrared range to make functional measurements of brain activity [2]. In recent years this modality has been investigated as part of a BCI application. Mental strategies used in most NIRS-BCI research includes either frontal lobe activation (mental arithmetic, verbal fluency) or motor cortex activation (imagined and overt limb movement). The advantage for using these strategies is that they require little or no user training to achieve adequate results. NIRS equipment is quite portable, non-invasive, and is considered safe for even long term neonatal monitoring [3].

\section{I-B. BCI Software Design}

All BCI software requires high levels of functionality, access to real-time data and the ability to rapidly build and test processing methods. Attempts have been made to standardize the software approaches in this research area [4], [5]. The most prolific is the work done by Schalk et al on the BCI2000 system [6].

Although these software systems were designed for modalities such as EEG and fMRI, the system described here is inspired by these approaches. This system implements proven methods of BCI processing to the relatively new area of fNIRS.

\section{BCI CONTROL SYSTEM}

In this section we discuss the software control system for a bespoke NIRS-BCI. Any control system must supply a high degree of functionality in the most convenient way to best investigate the possibilities of this modality. From a software perspective it is important to outline the software requirements which are then implemented according to priority. The National Instruments (NI) hardware that this system is comprised of is supplied with the LABVIEW development platform. This graphical programming and control utility is central to the robust and modular nature of the system design.

\section{II-A. Software Requirements}

An NIRS-BCI requires a varying range of functionality within its control system. Initially three essential requirements were specified.

- Uncomplicated hardware control and interfacing

- Software demodulation to replace a cumbersome hardware system

- The ability to rapidly implement and test real-time DSP and classification methods

Secondary requirements included integrated diagnostic capability, convenient raw data storage for offline analysis, 


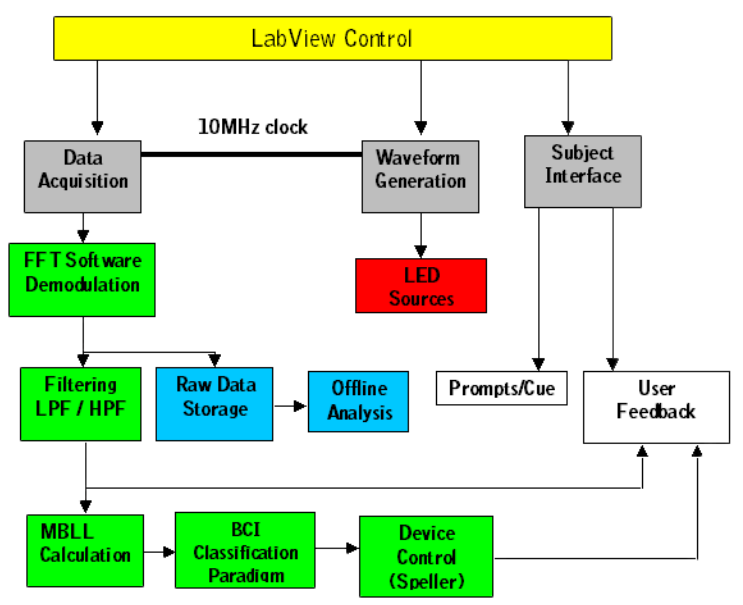

Fig. 1. Software Flow Diagram

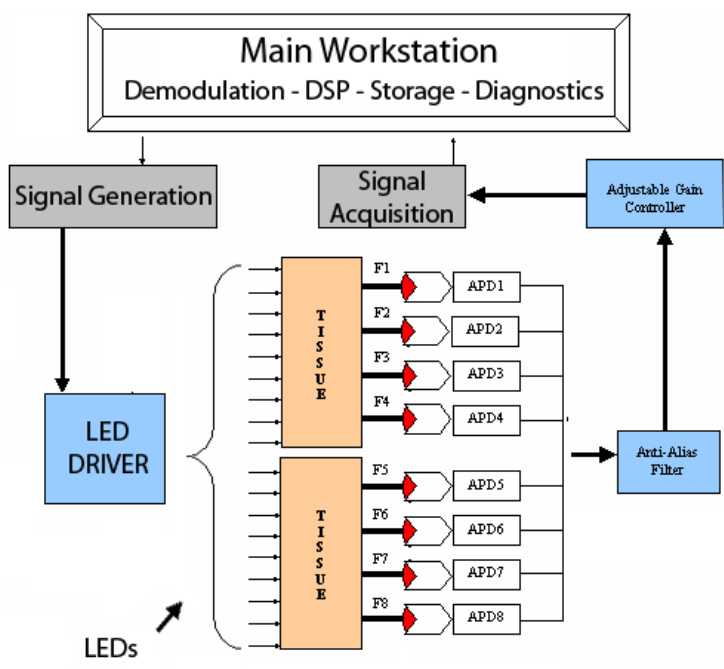

Fig. 2. Hardware Configuration experimental design control and aural subject cues. Firstly, it is necessary to examine the hardware system being controlled. Fig. 1 shows the conceptual flow diagram of the finished system.

\section{II-B. Hardware Configuration}

Data is acquired using two NI data acquisition cards (PXI-4462). These have four 24-bit analog input channels which are simultaneously sampled at up to $204 \mathrm{kHz}$. The modulation signals are generated using an NI 32-channel analog waveform generation card (PXI-6723). These three NI cards are housed in a PXI chassis (PXI-1033) which aids timing and synchronisation via the chassis backpane. This backpane allows timing and trigger information to be passed to all three cards. A custom LED driver was built to accurately modulate the triple wavelength LEDs. In a typical experiment, only two of these wavelengths $(760 \mathrm{~nm}$ and $880 \mathrm{~nm}$ ) are used. Avalanche photodiodes (APDs) are used to detect the resultant light intensity from the scalp. The PXI chassis is connected a desktop PC which controls the entire system. The hardware configuration can be seen in Figure 2.

\section{II-C. Hardware Control}

A significant advantage of basing this software on the LABVIEW platform is its dedicated hardware compatibility. Data acquisition and generation is performed with NI equipment and LABVIEW is designed to integrate seamlessly with their hardware. LABVIEW is also designed as general-purpose hardware-interaction software allowing the scope to introduce more acquisition and generation systems if the need arises.

The system investigated here uses LABVIEW routines to synchronise generation and acquisition for minimal phase distortion. A single front panel control system used by the researcher is designed to allow maximum flexibility at the start of each experiment. The researcher can control generation and sampling rates, the number of active LEDs and acquisitions channels, LED modulation frequencies and format of user feedback. The nature of the software design means the addition of LED and acquisition channels to the hardware system is trivial.

\section{II-D. Demodulation Strategies}

A key component to the system was the ability to discard an expensive and bulky hardware demodulation system. A difficulty of NIRS measurement is that the optical intensity is attenuated by about $10^{-8}$ during tissue interrogation. To allow the signals to be recovered, each wavelength is modulated by a carrier signal. In previous systems, separate hardware lock-in amplifiers were used to demodulate the signals [7]. This new system removes the need for expensive and cumbersome lock-in amplifiers and moves all demodulation into software [8]. Where previously the system was constrained by the requirement of these amplifiers, this new software alternative allows for more scalable and cost effective multichannel design. It also allows for investigation of as yet untested demodulation strategies.

Currently NIR light interrogates the region of interest from multiple LED sources. This light is then detected by a single APD. The APD's output is digitized and placed in the acquisition buffer for processing. This data, retrieved in windowed blocks, is processed with a Hanning-windowed FFT, producing a complex spectrum. This spectrum is passed to the frequency search function. The specific search frequencies are listed either in a text file or can be adjusted in the software prior to the experiment. The 
function also takes a search window for each frequency specified as a percentage of the sampling frequency.

This process can be repeated for any number of input channels constrained only by the processing time and physical hardware channels. The hardware of this system has eight usable input channels. Experiments have been conducted using three input channels with four dualwavelength LEDs per channel. Analysis of the processing time at this configuration gave no detrimental increase and implies that an eight channel configuration should pose no great difficulty to the system.

The output of the demodulation is the transmitted light levels for each LED wavelength. These are stored for further processing.

\section{II-E. Data Processing \& Classification}

A versatile system must be able to handle various data manipulation routines. An typical fNIRS signal is saturated with different types of physiological noise. Similar to the BCI classification approaches, there is no standard method of dealing with this corruption in fNIRS. A more detailed review of fNIRS noise and possible solutions is dealt with in [9]. Problems also arise when using a high number of channels and with the application of processing routines simultaneously to all channels. Potential loss of data and compromised hardware timing can result.

This system was designed to allow easy integration of the standard LABVIEW filter functions into the signal processing chain. User defined signal processing functions in $\mathrm{C}$ or $\mathrm{C}++$ can be integrated via a DLL or such functions can be written directly in LABVIEW and included in the processing chain. Most processing functions have been optimised for real-time operation. The system also enables the display of the timings for each process, leaving the researcher capable of calculating a time-budget allowed for each processing stage.

As with the flexibility of the signal processing functions, it is also possible to select from a number of different classification routines. Creating and including new classification schemes can be done using MATLAB, DLLs or LABVIEW code. A standard LABVIEW library includes a function allowing MATLAB source code files to be directly inserted into the control chain. This minimises development and test times, giving the researcher a quicker insight as to whether a function can adequately perform in real-time.

\section{II-F. Secondary Requirements}

\section{II-F.1. Test Systems}

The ability to analyse hardware and software performance and troubleshoot any problems that may arise is important for any software of this type. Included within this system is a diagnostic section that allows the researcher to inspect the raw signals coming from the system in real-time. It is possible to cycle through each data input channel and view the performance of LEDs and APDs. This tool is also used to check if source-detector positioning is appropriate and all channels are responding correctly before an experiment. II-F.2. Subject interaction and feedback

Prior to a simple activation-analysis experiment the researcher can decide the number and duration of stimulus and rest periods. It is possible to change the different cues for the subject depending on the interrogated area. Once the decision is made, the order of these cues are randomized for the subject. There is also a facility for the researcher to specify a timing range for randomized stimulus/rest durations. These cues delivered to the subject can be both visual and aural. All this is controlled within the same software platform as the previous sections. The timing clock used to synchronise the hardware is the same clock used to regulate stimulus periods.

Applications for $\mathrm{Hb} / \mathrm{HbO}$ feedback are also included and users can be encouraged to attempt to control the relative concentration changes through different mental tasks shown in Fig. 3. Future development will include some of the standard BCI demonstration applications like word spellers or very basic neuro-prosthesis control similar to [10].

\section{II-F.3. Data Storage}

At the end of each experiment the raw light intensity data is stored in a file for additional offline analysis. This data is also formatted into a file for use with the HomER NIRS analysis tool [11]. All data is timestamped with millisecond values from the hardware timing clock as well as a stimulus markers.

\section{EXPERIMENTAL RESULTS}

Currently the software is still in the testing stages of development. Initial experiments however have proven it to be quite powerful. Figure 3 shows the $\mathrm{Hb} / \mathrm{HbO}$ levels presented to a subject during an experiment. The user attempts to intentionally control these levels during an activation. Fig. 4 shows an set of activations from each channel and topographic map of the active area. This data was collected with the software during a motor cortex experiment and processed offline. These preliminary tests have successfully demonstrated the softwares basic functionality and convenience in dealing with NIRS signal.

\section{CONCLUSION}

The system achieves its primary and secondary requirements allowing maximum flexibility in its configuration for the researcher. Further development will attempt to include more BCI applications and standard DSP and classification routines.

\section{Acknowledgments}

Support for this work was provided by grants from Science Foundation Ireland (SFI). 


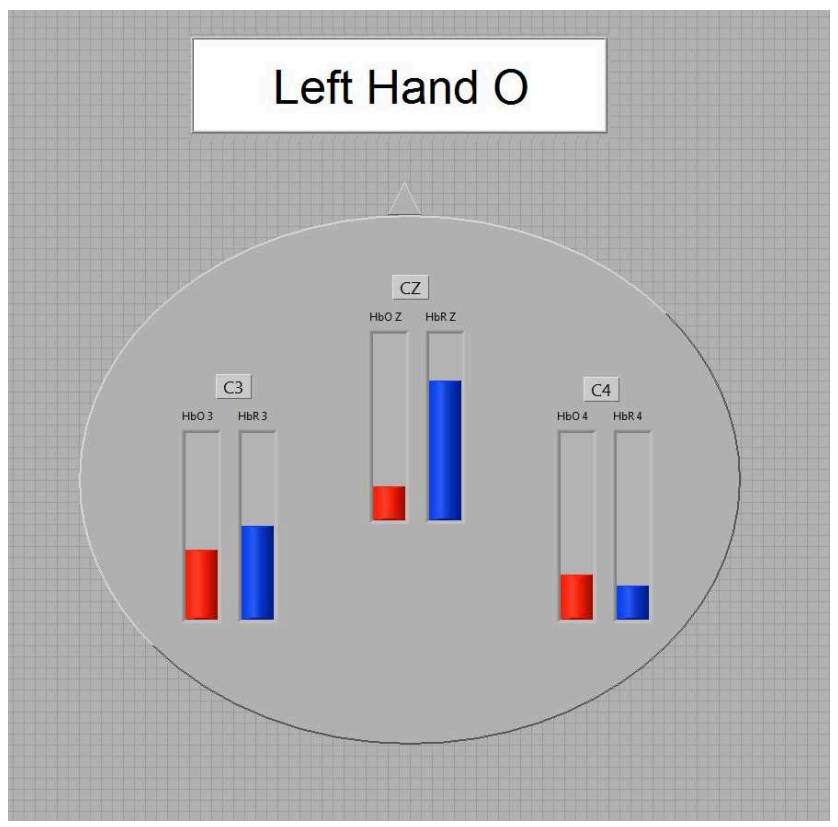

Fig. 3. Subject feedback screen. Subject can monitor localized motor cortex $\mathrm{Hb} / \mathrm{HbO}$ levels during activation.

\section{REFERENCES}

[1] F. F. Jobsis, "Noninvasive infrared monitoring of cerebral and myocardial oxygen sufficiency and circulatory parameters," Science, vol. 198, no. 4323, pp. 1264-7, 1977.

[2] A. Villringer, J. Planck, C. Hock, L. Schleinkofer, and U. Dirnagl, "Near infrared spectroscopy (NIRS): a new tool to study hemodynamic changes during activation of brain function in human adults," Neurosci Lett, vol. 154, no. 1-2, pp. 101-4, May 1993.

[3] A. Bozkurt and B. Onaral, "Safety assessment of near infrared light emitting diodes for diffuse optical measurements," Biomed Eng Online, vol. 3, no. 1, p. 9, 2004.

[4] L. Bianchi, F. Babiloni, F. Cincotti, S. Salinari, and M. Marciani, "Introducing bf++: A c++ framework for cognitive bio-feedback systems design," Meth. Inform. Med., vol. 42, no. 1, pp. 102-110, 2003.

[5] C. Guger, A. Schlogl, C. Neuper, D. Walterspacher, T. Strein, and G. Pfurtscheller, "Rapid prototyping of an eeg-based brain-computer interface (bci)," Neural Systems and Rehabilitation Engineering, IEEE Transactions on [see also IEEE Trans. on Rehabilitation Engineering], vol. 9, no. 1, pp. 49-58, Mar 2001.

[6] G. Schalk, D. McFarland, T. Hinterberger, N. Birbaumer, and J. Wolpaw, "Bci2000: a general-purpose brain-computer interface (bci) system," Biomedical Engineering, IEEE Transactions on, vol. 51, no. 6, pp. 1034-1043, June 2004.

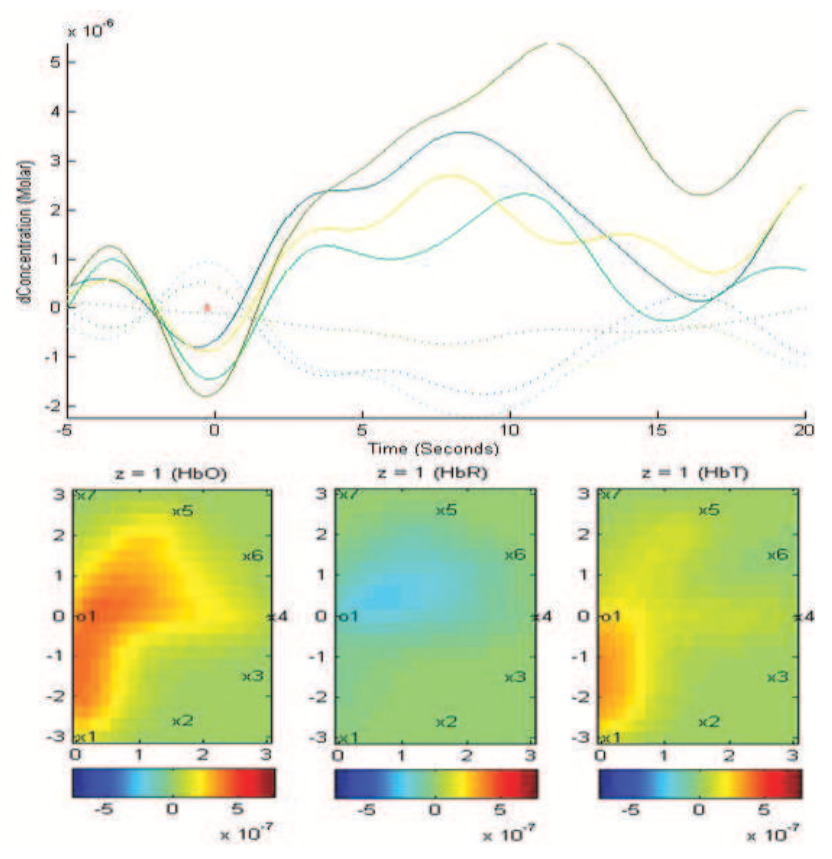

Fig. 4. Example of collected data, multi-channel data registering activation. Presented with HomER.

[7] S. Coyle, "Near-infrared spectroscopy for brain computer interfacing," Ph.D. dissertation, National University of Ireland, Maynooth, 2005.

[8] N. L. Everdell, A. P. Gibson, I. D. C. Tullis, T. Vaithianathan, J. C. Hebden, and D. T. Delpy, "A frequency multiplexed near-infrared topography system for imaging functional activation in the brain," Review of Scientific Instruments, vol. 76, no. 9, p. 093705, 2005. [Online]. Available: http://link.aip.org/link/?RSI/76/093705/1

[9] F. Matthews, B. Pearlmutter, T. Ward, C. Soraghan, and C. Markham, "Hemodynamics for braincomputer interfaces," Signal Processing Magazine, IEEE, vol. 25, no. 1, pp. 87-94, 2008.

[10] C. Soraghan, F. Matthews, D. Kelly, T. Ward, C. Markham, B. Pearlmutter, and R. O'Neill, "A dualchannel optical brain-computer interface in a gaming envrioment," in Proceedings of the 9th International Conference on Computer Games: AI, Animation, Mobile, Educational and Serious Games, Dublin Institute of Technology, Ireland, Nov. 2006.

[11] T. Huppert, "HomER nirs analysis software," 2006, this is an electronic document. Date retrieved: May 28, 2007. [Online]. Available: http://www.nmr.mgh.harvard.edu/PMI /resources/homer/home.htm 